\title{
Effect of hydraulic hysteresis on dynamic response of unsaturated soils
}

\author{
B. Shahbodagh ${ }^{\text {a }}$, N. Khalili, G. Alipour Esgandani \\ School of Civil and Environmental Engineering, The University of New South Wales, Sydney, NSW 2052, Australia
}

\begin{abstract}
Starting from the conservation laws, field equations governing the dynamic behaviour of unsaturated soils are presented. The coupling between solid and fluid phases is enforced according to the effective stress principle taking suction dependency and volume change of the effective stress parameters into account. The hydraulic hysteresis is accounted for through the effective stress parameter and the soil water characteristic curve. The spatial discretization of the governing equations is achieved using finite element method, whereas the time integration is conducted using the Newmark scheme. The numerical results are then presented, and the effect of hydraulic hysteresis on dynamic response of unsaturated soils is particularly emphasized.
\end{abstract}

\section{Introduction}

Despite the widespread distribution of unsaturated soils throughout the world, the development of constitutive and computational models for the dynamic behaviour of unsaturated soils has significantly lagged behind similar developments for dry and saturated soils. This has mainly been due to the inherent complexities associated with the dynamic behaviour of unsaturated soils, i.e. simultaneous flow of air and water through the porous materials, the presence of inertia forces in different phases, highly nonlinear deformation behaviour of the soil matrix, and complex interaction of fluid flow and deformation fields.

In recent years, several contributions have been made to dynamics of unsaturated soils. In the studies, two different approaches have generally been followed to enforce the coupling between solid and fluid phases. In the first approach, a single effective stress variable is used to describe the mechanical behaviour of unsaturated soils, e.g. see [1-7], while in the second approach two stress state variables, i.e. net stress and suction, are adopted to cast the constitutive relationships of the soil, e.g. see $[8,9]$. A major difficulty with the second approach is that it requires determination of two sets of material parameters, one for each of the stress state variables, which in fact may not be independent, and can lead to intractable stress strain relationships. In the effective stress based models developed for coupled dynamic analysis of unsaturated soils, the degree of saturation has mostly been used as the effective stress parameter. From experimental evidence, this parameter is strongly dependent on the soil structure and cannot be uniquely determined by the degree of saturation [10]. In addition, the effect of hydraulic hysteresis was ignored in the studies, which can significantly alter the response of unsaturated soils under dynamic loading conditions that

\footnotetext{
a Corresponding author: b.shahbodagh@unsw.edu.au
}

can invariably involve complex cycles of strain-induced wetting and drying.

In this paper, a coupled flow-deformation model is presented for the dynamic/seismic analysis of unsaturated soils. The theoretical approach adopted is based on the model proposed by Khalili et al. [11], addressing the suction dependency and volume change of the effective stress parameter. The effect of hydraulic hysteresis on the effective stress parameter and soil water characteristic curve is accounted for using the model proposed by Khalili and Zargarbashi [12]. The spatial discretization of the governing equations is achieved using finite element method, whereas the time integration is conducted using the Newmark technique. The numerical results are then presented, demonstrating the performance of the proposed approach, and the effect of hydraulic hysteresis on static and dynamic response of unsaturated soils is particularly emphasized.

\section{Basic Concepts}

\subsection{Effective Stress}

The concept of effective stress plays a key role in quantitative assessment of response in saturated and unsaturated soils. In its most common form, the effective stress can be expressed as [13]

$$
\sigma_{i j}^{\prime}=\sigma_{\text {net } i j}-\chi s \delta_{i j}
$$

where $\sigma_{\text {net } i j}=\sigma_{i j}+p_{G} \delta_{i j}$ is the net stress, $s=p_{G}-p_{W}$ is the matric suction, $\chi$ is the effective stress parameter, $\sigma_{i j}$ is the total stress, $p_{W}$ and $p_{G}$ are the pore water and pore gas pressures, respectively, and $\delta_{i j}$ is the Kronecker 
delta. The incremental form of the effective stress equation can be expressed as

$$
\dot{\sigma}_{i j}^{\prime}=\dot{\sigma}_{n e t ~ i j}-\psi \dot{s} \delta_{i j}
$$

where $\psi=\partial(\chi s) / \partial s$ is the incremental effective stress parameter, and superimposed dot denotes the time derivative with respect to the solid phase. Following the approach proposed by Khalili and Khabbaz [14] and Khalili et al. [15], the effective stress parameter is defined as

$$
\chi=\left\{\begin{array}{cl}
1 & \text { for } s \leq s_{e} \\
\left(s / s_{e}\right)^{-\Omega} & \text { for } s>s_{e}
\end{array}\right.
$$

where $\Omega$ is a material parameter with the best fit value of 0.55 , and $s_{e}$ is the suction value marking the transition between saturated and unsaturated states. For wetting process, $s_{e}$ is equal to the air expulsion value, $s_{e x}$, whereas for drying process, $s_{e}$ is equal to the air entry value, $s_{a e} \cdot s_{e}$ is a priori a function of the specific volume or volume change of the solid skeleton. This leads to a shift to the right of the effective stress parameter curve and soil water characteristic curve with increasing density, see Figure 1.

Khalili and Zargarbashi [12] experimentally studied the effect of hydraulic hysteresis on the effective stress parameter, and proposed the following relation for suction reversals:

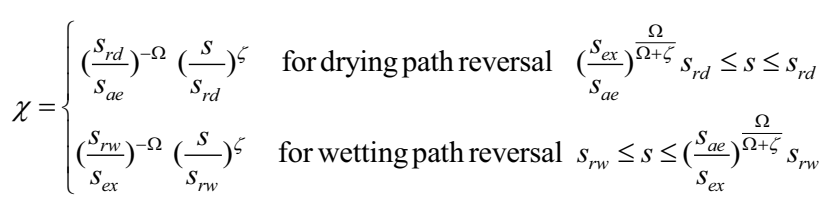

where $\zeta$ is the slope of the transition line between the main wetting and main drying paths in a $\ln \chi \sim \ln s$ plane, and $s_{r w}$ and $s_{r d}$ are the points of suction reversal on the main wetting and main drying paths, respectively.

\subsection{Soil Water Characteristic Curve}

Another important concept in the mechanics of unsaturated soils is the soil water characteristic curve (SWCC), which is used to determine the volumetric deformation of the water phase with respect to change in matric suction. In this formulation, the SWCC model proposed by Brooks and Corey [16], extended to include hydraulic hysteresis effect [11], is adopted as

$$
\begin{aligned}
& S_{e f f}=\left\{\begin{array}{cc}
1 & \text { for } s \leq s_{e} \\
\left(s_{e} / s\right)^{\lambda_{p}} & \text { for } s>s_{e}
\end{array}\right.
\end{aligned}
$$

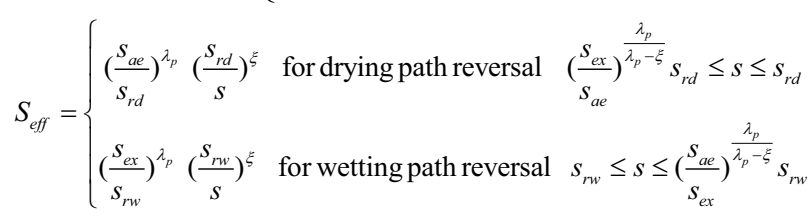

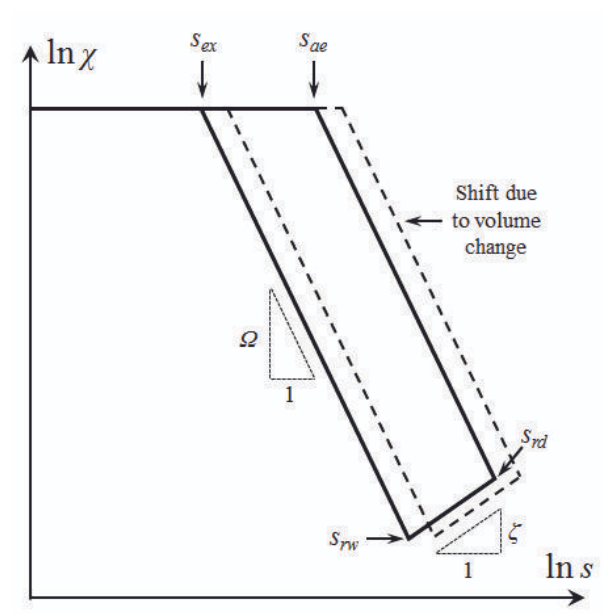

Figure 1. Evolution of the effective stress parameter $\chi$ with hydraulic hysteresis

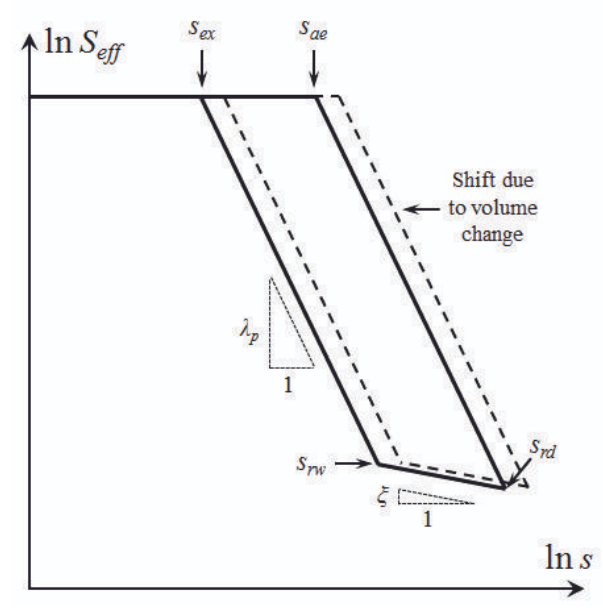

Figure 2. Soil water characteristic curve including hydraulic hysteresis

where $\lambda_{\mathrm{p}}$ is the pore size distribution index, $\mathrm{S}_{\mathrm{eff}}=\left(\mathrm{S}_{\mathrm{r}}\right.$ $\left.\mathrm{S}_{\text {res }}\right) /\left(1-\mathrm{S}_{\text {res }}\right)$ is the effective degree of saturation, and $\mathrm{S}_{\text {res }}$ is the residual degree of saturation, see Figure 2.

\section{Governing Equations}

\subsection{Conservation of Mass}

Unsaturated soils consist of three phases, i.e. solid (S), water (W), and gas (G), which within the context of theory of mixtures are assumed to be continuously distributed throughout representative elementary volume. By ignoring mass exchanges among the phases, the conservation of mass for each phase can be expressed by

$$
\frac{d_{\alpha}}{d t}\left(n^{\alpha} \rho_{\alpha}\right)+n^{\alpha} \rho_{\alpha} v_{\alpha i, i}=0 \quad(\alpha=S, W, G)
$$


where $v_{\alpha i}$ is the velocity vector of phase $\alpha$, and $d_{\alpha}\left(\mathrm{O} / d t=\partial\left(\mathrm{)} / \partial t+()_{, i} v_{\alpha i}\right.\right.$ is the material time derivative with respect to phase $\alpha$. The material time derivative of volume fraction of fluid phases $n^{\beta}(\beta=W, G)$ with respect to the solid phase is obtained as

$$
\frac{d_{S} n^{\beta}}{d t}=\frac{1}{V}\left(\frac{d_{S} V_{\beta}}{d t}-n^{\beta} \frac{d_{S} V}{d t}\right)=\frac{1}{V} \frac{d_{S} V_{\beta}}{d t}-n^{\beta} v_{S i, i}
$$

in which $V_{\alpha}$ is the volume of constituent $\alpha$, and $V$ is the total volume. Assuming barotropic fluid, and substituting (8) into (7), we obtain

$$
-\frac{1}{\rho_{\beta}}\left(\rho_{\beta} \dot{\omega}_{\beta i}\right)_{, i}=n^{\beta} c_{\beta} \frac{d_{S} p_{\beta}}{d t}+\frac{1}{V} \frac{d_{S} V_{\beta}}{d t}
$$

in which $\dot{\omega}_{\beta i}=n^{\beta}\left(v_{\beta i}-v_{S i}\right)$ is the relative velocity of fluid phases with respect to the solid phase, and $c_{\beta}$ is the compressibility coefficient for phase $\beta$. Invoking existence of elastic and plastic potentials, it can be shown that [11]

$$
\begin{gathered}
\frac{1}{V} \frac{d_{S} V_{W}}{d t}=\psi \dot{\varepsilon}_{v}+n \frac{\partial S_{r}}{\partial s} \dot{s} \\
\frac{1}{V} \frac{d_{S} V_{G}}{d t}=(1-\psi) \dot{\varepsilon}_{v}-n \frac{\partial S_{r}}{\partial s} \dot{s}
\end{gathered}
$$

Substituting (10)-(11) into (9), the mass balance equations for the water and gas phases, respectively, become

$$
\begin{gathered}
-\frac{1}{\rho_{W}}\left(\rho_{W} \dot{\omega}_{W i}\right)_{, i}=n^{W} c_{W} \frac{d_{S} p_{W}}{d t}+\psi \dot{\varepsilon}_{v}+n \frac{\partial S_{r}}{\partial s} \dot{s} \\
-\frac{1}{\rho_{G}}\left(\rho_{G} \dot{\omega}_{G i}\right)_{, i}=n^{G} c_{G} \frac{d_{S} p_{G}}{d t}+(1-\psi) \dot{\varepsilon}_{v}-n \frac{\partial S_{r}}{\partial s} \dot{s}
\end{gathered}
$$

\subsection{Conservation of Momentum}

The conservation of linear momentum of phase $\alpha$ gives

$$
\sigma_{j i, j}^{\alpha}+\rho_{\alpha} n^{\alpha} b_{i}+\sum_{\gamma \neq \alpha} h_{i}^{\alpha \gamma}=\rho_{\alpha} n^{\alpha} a_{\alpha i}
$$

where $\sigma_{i j}^{\alpha}$ is the partial Cauchy stress, $\gamma=S, W, G, b_{i}$ is the body force per unit mass, $h_{i}^{\alpha \gamma}\left(=-h_{i}^{\gamma \alpha}\right)$ is the interaction force per unit volume exerted by phase $\gamma$ on phase $\alpha$, and $a_{\alpha i}$ is the acceleration vector. Ignoring the relative accelerations between the phases and summing the conservation of momentum equations of the three phases yields

$$
\sigma_{j i, j}+\rho b_{i}=\rho a_{S i}
$$

where $\rho=\sum_{\alpha} \rho_{\alpha} n^{\alpha}$ is the density of the mixture, and $\rho_{\alpha}$ is the intrinsic density of phase $\alpha$.
From the incorporation of the momentum balance equations into the mass balance equations, the continuity equations for the fluid phases become

$$
\begin{aligned}
& \frac{1}{\rho_{W}}\left(\frac { k _ { W } } { g } \left(p_{W, i}-\right.\right.\left.\left.\rho_{W} b_{i}+\rho_{W} a_{S i}\right)\right)_{, i} \\
&=n^{W} c_{W} \frac{d_{S} p_{W}}{d t}+\psi \dot{\varepsilon}_{v}+n \frac{\partial S_{r}}{\partial s} \dot{s} \\
& \frac{1}{\rho_{G}}\left(\frac{k_{G}}{g}\left(p_{G, i}-\rho_{G} b_{i}+\rho_{G} a_{S i}\right)\right)_{, i} \\
&=n^{G} c_{G} \frac{d_{S} p_{G}}{d t}+(1-\psi) \dot{\varepsilon}_{v}-n \frac{\partial S_{r}}{\partial s} \dot{s}
\end{aligned}
$$

\subsection{Constitutive Equations}

Constitutive relationships for volumetric deformations of fluid phases can be expressed as [11]

$$
\begin{gathered}
\frac{\dot{V}_{W}}{V}=\psi \dot{\varepsilon}_{v}+a_{W} \dot{s} \\
\frac{\dot{V}_{G}}{V}=(1-\psi) \dot{\varepsilon}_{v}+a_{G} \dot{s}
\end{gathered}
$$

which relates the changes in pore fluid volumes to rates of suction and volumetric strain $\dot{\varepsilon}_{v}$. Due to highly nonlinear behaviour of soils, the stress-strain relationship of the soil skeleton is written in the incremental format as

$$
\dot{\sigma}_{i j}^{\prime}=C_{i j k l} \dot{\varepsilon}_{k l}
$$

where $C_{i j k l}$ is the constitutive tensor, and $\dot{\varepsilon}_{k l}$ is the soil skeleton strain tensor. The constitutive model adopted to express $C_{i j k l}$ must be able to explain the deformation characteristics of the soil skeleton under cyclic loading conditions [11, 17-18].

\section{Spatial and Temporal Discretization}

For a quantitative solution, the weak forms of the equations of motion and flow continuity equations are obtained by applying the Galerkin method and spatially discretized using the finite element procedure. The primary unknown variables in the whole domain can be expressed in terms of the nodal values as

$$
\{u\}=[N]\left\{u_{N}\right\}, p_{\beta}=\left\{N_{h}\right\}^{T}\left\{p_{\beta N}\right\}
$$

where $[N]$ and $\left\{N_{h}\right\}$ represent the displacement shape functions for the solid phase, and the shape functions for the pore fluid pressures, respectively. The spatially discretized form of the governing partial differential equations can be written as

$$
\begin{aligned}
{[M]\left\{\ddot{u}_{N}\right\} } & +[K]\left\{\Delta u_{N}\right\}-\chi\left[K_{v}\right]\left\{p_{W N}\right\} \\
& -(1-\chi)\left[K_{v}\right]\left\{p_{G N}\right\}=\{F\}-\{R\}_{t}
\end{aligned}
$$




$$
\begin{gathered}
\rho_{W} \tilde{k}^{W}\left[K_{v}\right]^{T}\left\{\ddot{u}_{N}\right\}-\psi\left[K_{v}\right]^{T}\left\{\dot{u}_{N}\right\}-\tilde{k}^{W}\left[K_{h}\right]\left\{p_{W N}\right\} \\
-c_{11}\left[K_{n}\right]\left\{\dot{p}_{W N}\right\}+c_{12}\left[K_{n}\right]\left\{\dot{p}_{G N}\right\}=-\tilde{k}^{W}\left\{q^{W}\right\} \\
\rho_{G} \tilde{k}^{G}\left[K_{v}\right]^{T}\left\{\ddot{u}_{N}\right\}-(1-\psi)\left[K_{v}\right]^{T}\left\{\dot{u}_{N}\right\}-\tilde{k}^{G}\left[K_{h}\right]\left\{p_{G N}\right\} \\
-c_{22}\left[K_{n}\right]\left\{\dot{p}_{G N}\right\}+c_{12}\left[K_{n}\right]\left\{\dot{p}_{W N}\right\}=-\tilde{k}^{G}\left\{q^{G}\right\}
\end{gathered}
$$

where $\quad c_{11}=n^{W} c_{W}-n \partial S_{r} / \partial s, \quad c_{22}=n^{G} c_{G}-n \partial S_{r} / \partial s$, $c_{12}=-n \partial S_{r} / \partial s, \quad \tilde{k}^{\beta}=k^{\beta} / \rho_{\beta} g,[M]$ is the mass matrix, $[K]$ is the element stiffness matrix, $\left[K_{v}\right]$ is the coupling matrix, $\left[K_{h}\right]$ is the flow matrix, $\left[K_{n}\right]$ is the pore mass matrix, $\left\{q^{W}\right\}$ and $\left\{q^{G}\right\}$ are vectors of nodal fluxes of the water and gas flows, respectively, and $\{F\}$ and $\{R\}_{t}$ are the vectors of nodal forces. The definition of the finite element matrices can be found in [19]. The Newmark scheme is used for the time integration of the discretized governing equations, with the lowest allowable order for each primary variable. The unconditional stability of the Newmark scheme is achieved when $2 \beta \geq \gamma \geq 0.5$ in which $\beta$ and $\gamma$ are the Newmark's parameters.

\section{Numerical Examples}

For the numerical analysis, a boundary value problem consisting of an unsaturated porous medium of $10 \mathrm{~m}$ height and $10 \mathrm{~m}$ width with an initial suction of $20 \mathrm{kPa}$ is considered, see Figure 3. The mesh is composed of 400 mixed quadrilateral elements of dimensions $0.5 \mathrm{~m} \times 0.5 \mathrm{~m}$. The upper boundary is drained and partially subjected to a load of width $B=3.0 \mathrm{~m}$ with a uniform intensity $f(\tau)$, while the remaining boundaries are impervious. As the displacement boundary, the side boundaries are supported horizontally, and the bottom surface is vertically constrained. Table 1 shows the material parameters adopted for the analyses. The parameters for the fully saturated state are the same as those used by Simon et al. [20]. For the case with hydraulic hysteresis effect, the air entry and air expulsion values of $\mathrm{s}_{\mathrm{ae}}=10 \mathrm{kPa}, \mathrm{s}_{\mathrm{ex}}=5 \mathrm{kPa}$, along with $\xi=0.04$, and $\zeta=0.15$ are used in the simulations, whereas for the case without hysteresis effect $s_{a e}=s_{e x}=10 \mathrm{kPa}$ are adopted. The initial suction is assumed to be placed on the main drying path. In the case with hydraulic hysteresis, wetting and drying occurs along the scanning and main drying paths, respectively, whereas without hysteresis the wetting-drying cycles occur along the main drying path. The analyses are performed under a harmonic loading condition with angular frequency $\omega=62.83 \mathrm{rad} / \mathrm{sec}$. A comparison of numerical results of the proposed hysteretic approach with those of the non-hysteretic approach is demonstrated in Figure 4 where $\hat{u}\left(=u V_{p} \gamma_{W} s_{I n i} /\left(k_{W S} \sigma_{\max } s_{a e}\right)\right)$ is the normalized displacement and $\tau\left(=t \gamma_{W} /\left(\rho_{s a t} k_{W s}\right)\right)$ is the normalized time. By taking the effect of hydraulic hysteresis into account, a deviation of the center line of

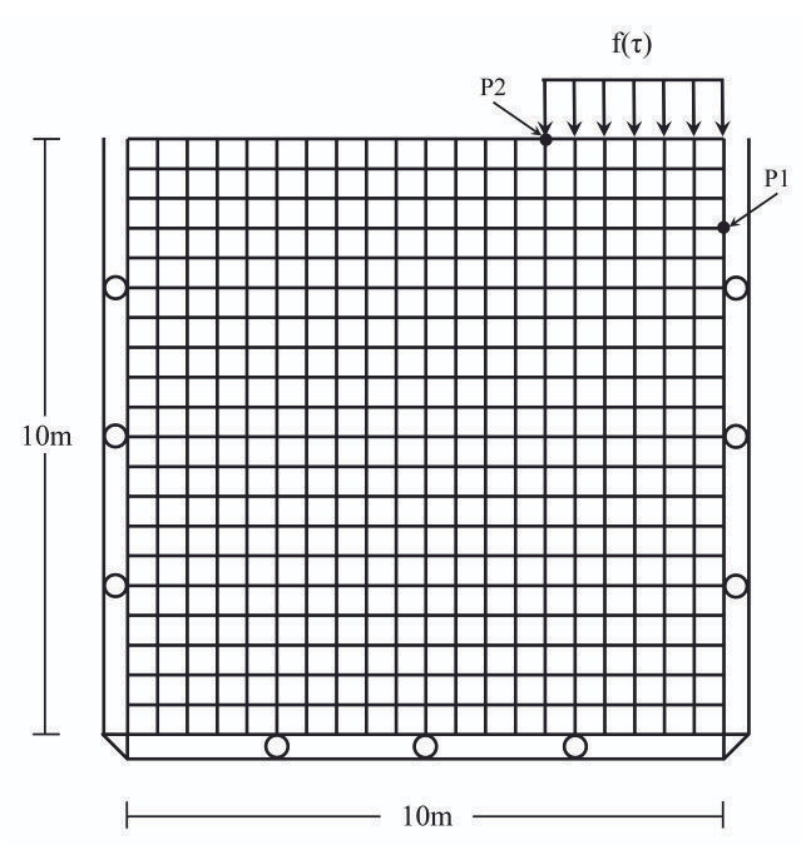

Figure 3. Finite element mesh and boundary conditions

the response from $\hat{u}=0$ line is observed. Also, considering the hydraulic hysteresis in the analysis induces significantly larger variations in suction level during dynamic loadings.

\section{Conclusions}

A rigorous framework based on the theory of mixtures is presented for describing the dynamic behaviour of unsaturated soils. The coupling between solid and fluid phases is established through the effective stress principle taking suction dependency and volume change of the effective stress parameter into account. The hydraulic hysteresis is accounted for through the effective stress parameter and the soil water characteristic curve. The change in the degree of saturation due to a change in the volume of the pore at constant suction is also taken into account. The numerical results demonstrate that the hydraulic hysteresis markedly alter the response of unsaturated soils under dynamic loading condition.

Table 1. Material parameters considered for the analysis.

\begin{tabular}{|lc|c|}
\hline Initial void ratio & $e_{0}$ & 0.5 \\
\hline Lame's constant $(\mathrm{kPa})$ & $\lambda$ & 833.3 \\
\hline Lame's constant $(\mathrm{kPa})$ & $\mu$ & 1250.0 \\
\hline Permeability of liquid at $\mathrm{S}_{\mathrm{r}}=1(\mathrm{~m} / \mathrm{s})$ & $k_{W s}$ & 0.01425 \\
\hline Permeability of gas at $\mathrm{S}_{\mathrm{r}}=0(\mathrm{~m} / \mathrm{s})$ & $k_{G s}$ & 0.05 \\
\hline Density of saturated mixture $\left(t / \mathrm{m}^{3}\right)$ & $\rho_{\text {sat }}$ & 1.8 \\
\hline Pore size distribution index & $\lambda_{p}$ & 0.15 \\
\hline Residual degree of saturation & $S_{r e s}$ & 0.2 \\
\hline
\end{tabular}



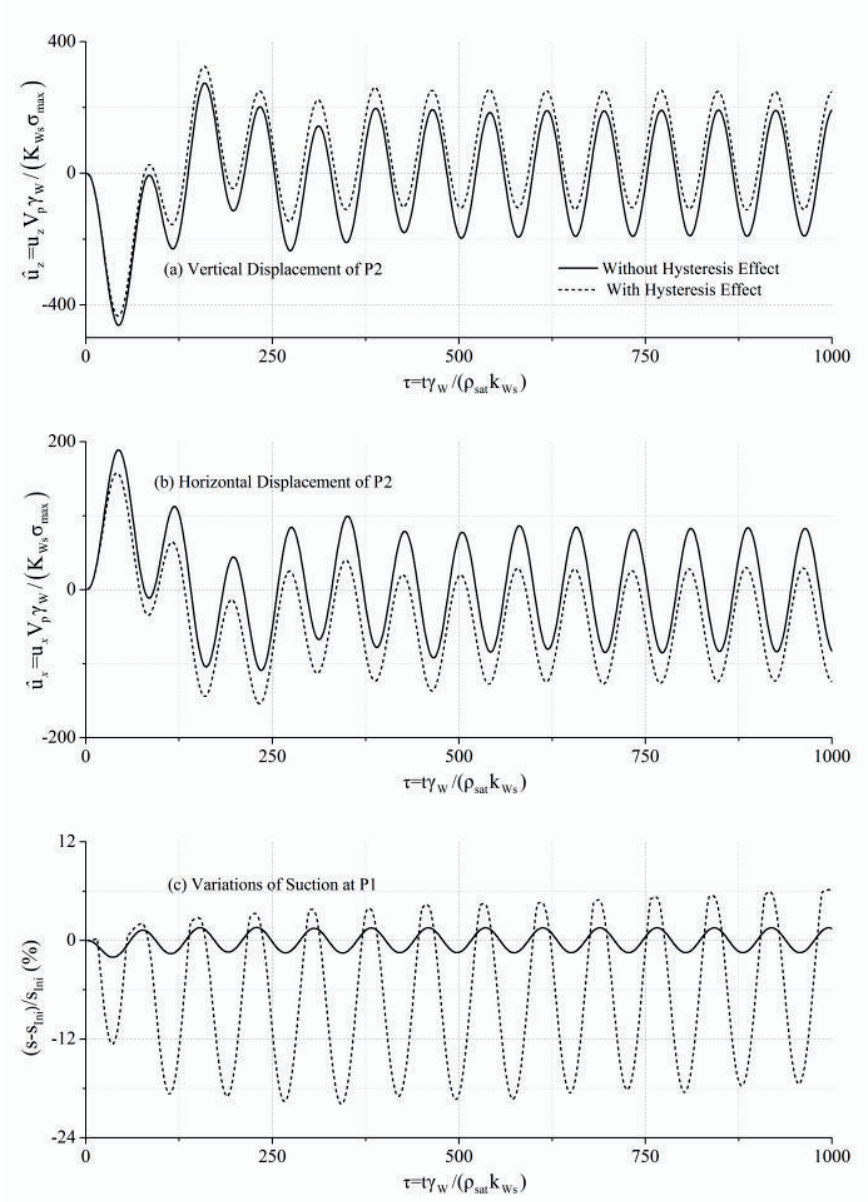

Figure 4. Effect of hydraulic hysteresis on dynamic response of the unsaturated porous medium

\section{References}

[1] Zienkiewicz OC, Xie YM, Schrefler BA, Ledesma A, Biĉaniĉ N. Static and dynamic behaviour of soils: a rational approach to quantitative solutions. II. Semi-saturated problems. Proceedings of the Royal Society of London. Series A, Mathematical and Physical Sciences 1990; 429: 311-321.

[2] Meroi EA, Schrefler BA, Zienkiewicz OC. Large strain static and dynamic semisaturated soil behaviour. International Journal for Numerical and Analytical Methods in Geomechanics 1995; 19: 81106.

[3] Schrefler BA, Scotta R. A fully coupled dynamic model for two-phase fluid flow in deformable porous media. Computer Methods in Applied Mechanics and Engineering 2001; 190: 3223-3246.

[4] Shahbodagh Khan B. Large deformation dynamic analysis method for partially saturated elastoviscoplastic soils, $P h D$ dissertation, Kyoto University, 2011.

[5] Uzuoka R, Borja RI. Dynamics of unsaturated poroelastic solids at finite strain. International Journal for Numerical and Analytical Methods in Geomechanics 2012; 36: 1535-1573.

[6] B Shahbodagh, G Esgandani, N Khalili. Large deformation dynamic analysis of unsaturated soils.
The 6th International Conference on Unsaturated Soils, UNSAT 2014, Sydney, 2014; 755 - 760.

[7] B Shahbodagh, N Khalili. A u-p formulation for fully coupled dynamic analysis of flow and deformation in unsaturated soils. The 2nd Australasian Conference on Computational Mechanics, Brisbane, 2015.

[8] Muraleetharan KK, Wei C. Dynamic behaviour of unsaturated porous media: governing equations using the Theory of Mixtures with Interfaces (TMI). International Journal for Numerical and Analytical Methods in Geomechanics 1999; 23: 1579-1608.

[9] Ravichandran N, Muraleetharan KK. Dynamics of unsaturated soils using various finite element formulations. International Journal for Numerical and Analytical Methods in Geomechanics 2009; 33: 611-631.

[10] Zerhouni MI. Application des réseaux de Petri continus à l'analyse dynamique des systèmes de production. Thèse de Doctorat de l'INP Grenoble, France; 1991.

[11] Khalili N, Habte MA, Zargarbashi S, A fully coupled flow deformation model for cyclic analysis of unsaturated soils including hydraulic and mechanical hystereses, Computers and Geotechnics 2008; 35(6): 872-889.

[12] Khalili N, Zargarbashi S. Influence of hydraulic hysteresis on effective stress in unsaturated soils. Geotechnique 2010; 60(9): 729-739.

[13] Bishop, A.W. 1959. The principle of effective stress. Teknisk Ukeblad;106(39): 859-863.

[14] Khalili N, and Khabbaz MH. A unique relationship for shear strength determination of unsaturated soils, Geotechnique 1998; 48(5): 681-688.

[15] Khalili N., Geiser F, Blight G. Effective Stress in Unsaturated Soils: Review with New Evidence. International Journal of Geomechanics 2004; 4(2): 115-126.

[16] Brooks RH, Corey AT. Hydraulic properties of porous media: Hydrology Papers. Colorado State University, Colorado, 1964.

[17] Oka F, Kimoto S. Computational modeling of multiphase geomaterials. CRC press, Taylor \& Francis group, Boca Raton, London and New York, 2012.

[18] Kimoto S, Shahbodagh Khan B, Mirjalili M, Oka F. A cyclic elasto-viscoplastic constitutive model for clay considering the nonlinear kinematic hardening rules and the structural degradation, International Journal of Geomechanics 2013; 15(5), A4014005.

[19] Shahbodagh Khan, B., Mirjalili, M., Kimoto, S. and Oka, F. 2013. Dynamic analysis of strain localization in water-saturated clay using a cyclic elasto-viscoplastic model, International Journal for Numerical and Analytical Methods in Geomechanics 2014; 38(8), 771-793.

[20] Simon BR, Zienkiewicz OC, Paul DK. An analytical solution for the transient response of saturated porous elastic solids. International Journal for Numerical and Analytical Methods in Geomechanics 1984; 8: 381-398. 